\title{
Novel Approach for Dealing with Partial Differential Equations with Mixed Derivatives
}

\author{
Abdon Atangana ${ }^{1}$ and Suares Clovis Oukouomi Noutchie ${ }^{2}$ \\ ${ }^{1}$ Institute for Groundwater Studies, Faculty of Natural and Agricultural Sciences, University of the Free State, \\ Bloemfontein 9301, South Africa \\ ${ }^{2}$ Department of Mathematical Sciences, North-West University, Mafikeng Campus, Mmabatho 2735, South Africa
}

Correspondence should be addressed to Abdon Atangana; abdonatangana@yahoo.fr

Received 25 March 2014; Revised 2 May 2014; Accepted 2 May 2014; Published 22 May 2014

Academic Editor: Ali H. Bhrawy

Copyright (c) 2014 A. Atangana and S. C. Oukouomi Noutchie. This is an open access article distributed under the Creative Commons Attribution License, which permits unrestricted use, distribution, and reproduction in any medium, provided the original work is properly cited.

We propose a powerful iteration scheme for solving analytically a class of partial equations with mixed derivatives. Our approach is based upon the Lagrange multiplier in two-dimensional spaces. The local convergence and uniqueness of the proposed method are analyzed. In order to demonstrate the applicability of our method, we present an algorithm to compute the solution for two examples.

\section{Introduction}

In the recent decade, several scholars in the fields of partial differential equations have paid attention in showing the existence and the solutions of the class of partial differential equations involving mixed and nonmixed derivatives. Several methods were proposed, for instance, the Laplace transform method [1-3], the Mellin transform method [4], the Fourier transform method $[5,6]$, and the Sumudu transform method [7-9] and the Green function method [10] for linear cases. Perturbation method [11], variational iteration method [1214], homotopy decomposition and perturbation method [1518], and others were developed for both linear and nonlinear cases.

While doing a search in the literature, we noticed that there is a class of partial differential equations for which no analytical method or iteration method has been proposed to get to the bottom of their solutions. Without loss of generality, the general form of this class of equation is given below as

$$
\begin{gathered}
\partial_{x^{n}}^{n} \partial_{y^{m}}^{m} \cdots \partial_{t^{i}}^{i}[U(x, y, \ldots, t)]+L[U(x, y, \ldots, t)] \\
+N[U(x, y, \ldots, t)]=f(x, y, \ldots, t),
\end{gathered}
$$

where, $m, n, \ldots, i$ are natural numbers, $L$ and $N$ are linear and nonlinear operators with only mixed derivatives, respectively, and $f$ is a known function. It is perhaps important to mention that proving the existence of a partial differential equation may be a very difficult task but it is only useful in pure mathematics. However, while dealing with real world problem, one needs to present the numerical or analytical solution because the proof of existence is not worth in this case. In order to satisfy scholars that deal with real world problems, several analytical methods have been developed in the recent decade. Nevertheless, we are afraid to say that those methods are not powerful enough to handle the above equation because of its complexity.

In this paper, our approach will be based upon the Lagrange multiplier in two-dimensional spaces. The local convergence and uniqueness of the proposed method will be analyzed in detail.

\section{Method for Solution}

We devote this section to the discussion underpinning the general method to derive the special solution of (1). The foremost item of the technique is as follows: the solution of a mathematically real world problem with linearization 
postulation is used as an initial guesstimate; formerly an additional extremely detailed estimate at some special point can be gotten.

We will assume that $H(x, y, \ldots, t)$ is the solution of the linear part of (1); we can record an illustration to appropriate the value of the selected singular point, for example, at $X(x, y, \ldots, t)$, and then the corrected solution can be written as follows:

$$
\begin{aligned}
& U(\alpha, \beta, \ldots, \tau)= H(\alpha, \beta, \ldots, \tau) \\
&+\int_{0}^{\alpha} \ldots \int_{0}^{\tau} \lambda(x, y, \ldots, t) \\
& \quad \times\left(\partial_{x^{n}}^{n} \partial_{y^{m}}^{m} \cdots \partial_{t^{i}}^{i}[U(x, y, \ldots, t)]\right. \\
&+L[U(x, y, \ldots, t)] \\
&+N[U(x, y, \ldots, t)] \\
&\quad-f(x, y, \ldots, t)) d x \cdots d t
\end{aligned}
$$

We will point out that $\lambda(x, y, \ldots, t)$ is the Lagrange multiplier [12] and the second term on the right is called the correction. The method has been modified into an iteration method [48 ] in the following approach:

$$
\begin{aligned}
& U_{n+1}(\alpha, \beta, \ldots, \tau) \\
& =H(\alpha, \beta, \ldots, \tau) \\
& +\int_{0}^{\alpha} \cdots \int_{0}^{\tau} \lambda(x, y, \ldots, t) \\
& \quad \times\left(\partial_{x^{n}}^{n} \partial_{y^{m}}^{m} \ldots \partial_{t^{i}}^{i}\left[U_{n}(x, y, \ldots, t)\right]\right. \\
& \quad+L\left[U_{n}(x, y, \ldots, t)\right] \\
& \quad+N[\widetilde{U}(x, y, \ldots, t)] \\
& \quad-f(x, y, \ldots, t)) d x \cdots d t
\end{aligned}
$$

besides $H(\alpha, \beta, \ldots, \tau)$ as preliminary guesstimate with likelynonentities and $\widetilde{U}(x, y, \ldots, t)$ is pondered as a circumscribed adaptation meaning $\delta \widetilde{U}(x, y, \ldots, t)=0$. Indeed for random $(\alpha, \beta, \ldots, \tau)$, the above equation can be reformulated as follows:

$$
\begin{aligned}
& U_{n+1}(X, Y, \ldots, T) \\
& =H(X, Y, \ldots, T) \\
& +\int_{0}^{X} \ldots \int_{0}^{T} \lambda(x, y, \ldots, t) \\
& \quad \times\left(\partial_{x^{n}}^{n} \partial_{y^{m}}^{m} \ldots \partial_{t^{i}}^{i}\left[U_{n}(x, y, \ldots, t)\right]\right. \\
& \quad+L\left[U_{n}(x, y, \ldots, t)\right] \\
& \quad+N[\widetilde{U}(x, y, \ldots, t)] \\
& \quad-f(x, y, \ldots, t)) d x \cdots d t .
\end{aligned}
$$

For straight problems, its exact answer can be achieved via one repetition step because of the statement that the Lagrange multiplier can be faithfully acknowledged.

We will in the coming section illustrate this extension by solving some problems with mixed derivatives. However, we will first deal with the convergence and uniqueness analysis of a specific equation (5).

\section{Convergence Analysis of the Iteration Method}

The purpose of this section is to show the local convergence of the proposed method for solving an example of nonlinear equation and the uniqueness of the special solution obtained via the proposed method; we will therefore consider the following equation:

$$
\begin{aligned}
\partial_{x t}^{2} u+ & 2 u \partial_{x^{2} t^{2}}^{4} u+4 \partial_{x} u \partial_{x t^{2}}^{3} u+4 \partial_{x^{2} t}^{3} u \partial_{t} u \\
& +4\left(\partial_{x t}^{2} u\right)^{2}+\partial_{t^{2}}^{2} u \partial_{x^{2}}^{2} u+u^{2}+u=0 .
\end{aligned}
$$

Let us consider the equation in the Hilbert space $\mathscr{H}=$ $L^{2}((\eta, \lambda) \times[0, T])$, defined as

$$
\mathscr{H}=\left\{(u, v):(\eta, \lambda) \times[0, T] \text { with, } \int u v d \iota d \kappa<\infty\right\} .
$$

Then, the operator is of the form

$$
\begin{aligned}
T(u)= & \partial_{x t}^{2} u+2 u \partial_{x^{2} t^{2}}^{4} u+4 \partial_{x} u \partial_{x t^{2}}^{3} u \\
& +4 \partial_{x^{2} t}^{3} u \partial_{t} u+4\left(\partial_{x t}^{2} u\right)^{2}+\partial_{t^{2}}^{2} u \partial_{x^{2}}^{2} u \\
& +u^{2}+u .
\end{aligned}
$$

The proposed analytical method is convergent if the following requirements are met.

Hypothesis 1. It is possible for us to find a positive constant say $F$ such that the inner product satisfies the following condition in $\mathscr{H}$ :

$$
(T(u)-T(v), u-v) \leq F\|u-v\|, \quad \forall v, u \in \mathscr{H} .
$$

Hypothesis 2. To the extent that all $v, u \in H$ are bounded implying that we can find a positive constant say $C$ such that $\|u\|,\|v\| \leq C$, then we can find $\Phi(C)>0$ such that

$$
(T(u)-T(v), g) \leq \Phi(C)\|u-v\|\|g\|, \quad \forall g \in H .
$$

We can consequently state the resulting theorem for the sufficient condition of the convergence of iteration method for (5).

Theorem 1. Let us consider

$$
\begin{aligned}
T(u)= & \partial_{x t}^{2} u+2 u \partial_{x^{2} t^{2}}^{4} u+4 \partial_{x} u \partial_{x t^{2}}^{3} u \\
& +4 \partial_{x^{2} t}^{3} u \partial_{t} u+4\left(\partial_{x t}^{2} u\right)^{2}+\partial_{t^{2}}^{2} u \partial_{x^{2}}^{2} u \\
& +u^{2}+u
\end{aligned}
$$

and consider the initial and boundary conditions for (5); then the proposed method leads to a special solution of (5). 
We will present the proof of this theorem by just verifying the Hypotheses 1 and 2.

Proof. Using the definition of our operator $T$, we have the following:

$$
\begin{aligned}
T(u)-T(v)= & \partial_{x t}^{2}(u-v)+2 u \partial_{x^{2} t^{2}}^{4} u+4 \partial_{x} u \partial_{x t^{2}}^{3} u \\
& +4 \partial_{x^{2} t}^{3} u \partial_{t} u+4\left(\partial_{x t}^{2} u\right)^{2}+\partial_{t^{2}}^{2} u \partial_{x^{2}}^{2} u \\
& +(u-v)^{2}+(u-v)-2 v \partial_{x^{2} t^{2}}^{4} v \\
& -4 \partial_{x} v \partial_{x t^{2}}^{3} v-4 \partial_{x^{2} t^{2}}^{3} v \partial_{t} v \\
& -4\left(\partial_{x t}^{2} v\right)^{2}-\partial_{t^{2}}^{2} v \partial_{x^{2}}^{2} v \\
T(u)-T(v)= & \partial_{x t}^{2}(u-v)+(u-v)^{2}+(u-v) \\
& +2 \partial_{x}\left(u \partial_{x t^{2}}^{3} u+\partial_{x t}^{2} u \partial_{t} u+\partial_{t x}^{2} u \partial_{t} u+\partial_{x} u \partial_{t^{2}}^{2} u\right) \\
& -2 \partial_{x}\left(v \partial_{x t^{2}}^{3} v+\partial_{x t}^{2} v \partial_{t} v+\partial_{t x}^{2} v \partial_{t} v+\partial_{x} v \partial_{t^{2}}^{2} v\right) \\
T(u)-T(v)= & \partial_{x t}^{2}(u-v)+\left(u^{2}-v^{2}\right) \\
& +(u-v)+\partial_{x^{2} t^{2}}^{4} u-\partial_{x^{2} t^{2}}^{4} v \\
T(u)-T(v)= & \partial_{x t}^{2}(u-v)+\left(u^{2}-v^{2}\right) \\
& +(u-v)+\partial_{x^{2} t^{2}}^{4}\left(u^{2}-v^{2}\right) .
\end{aligned}
$$

With the above reduction in hand, it is therefore possible for us to evaluate the following inner product:

$$
\begin{aligned}
& (T(u)-T(v),(u-v)) \\
& \quad\left(\partial_{x t}^{2}(u-v), u-v\right)+\left(\left(u^{2}-v^{2}\right), u-v\right) \\
& \quad+((u-v), u-v)+\left(\partial_{x^{2} t^{2}}^{4}\left(u^{2}-v^{2}\right), u-v\right) .
\end{aligned}
$$

We will examine case after case starting with

$$
\left(\partial_{x t}^{2}(u-v), u-v\right)
$$

Assuming that $u, v$ are bounded, therefore we can find a positive constant $M$ such that $(u, u),(v, v)<M^{2}$. It follows by the use of Schwartz inequality that

$$
\left(\partial_{x t}^{2}(u-v), u-v\right) \leq\left\|\partial_{x t}^{2}(u-v)\right\|\|u-v\| \text {. }
$$

However, we can find a positive constant $\omega$ such that $\left\|(u-v)_{x}\right\| \leq \omega\|u-v\|$; it follows from (14) that

$$
\left(\partial_{x t}^{2}(u-v), u-v\right) \leq \omega_{1} \omega_{2}\|u-v\|^{2}
$$

Also, we have the following inequality

$$
\begin{aligned}
& \left(u^{2}-v^{2}, u-v\right) \leq\left\|u^{2}-v^{2}\right\|\|u-v\| \leq \theta_{1} \theta_{2}\|u-v\|^{2} \\
& ((u-v), u-v) \leq\|u-v\|^{2} .
\end{aligned}
$$

We also have moreover that the Cauchy-Schwarz-Bunyakovsky inequality yields

$$
\left(\partial_{x^{2} t^{2}}^{4}\left(u^{2}-v^{2}\right), u-v\right) \leq \theta_{3} \theta_{4} \theta_{5} \theta_{6}\left\|u^{2}-v^{2}\right\|\|u-v\| .
$$

Obviously due to the fact that it is possible for us to find two positive constants $\theta_{3}, \theta_{4}$ such that

$$
\partial_{x^{2} t^{2}}^{4}\left(\left(u^{2}-v^{2}\right), u-v\right) \leq \theta_{3} \theta_{4}\left\|\left(u^{2}-v^{2}\right)_{x t}\right\|\|u-v\|,
$$

then we can find another set of positive constants $\theta_{5} \theta_{6}$ respecting the following inequality:

$$
\left\|\left(u^{2}-v^{2}\right)_{x t}\right\| \leq \theta_{5} \theta_{6}\left\|u^{2}-v^{2}\right\|
$$

and finally we can find two positive constants $\theta_{7}$ and $\theta_{8}$ verifying

$$
\left(\partial_{x^{2} t^{2}}^{4}\left(u^{2}-v^{2}\right), u-v\right) \leq \theta_{3} \theta_{4} \theta_{5} \theta_{6} \theta_{7} \theta_{8}\|u-v\|^{2} .
$$

Now, substituting (20), (16), and (15) into (12) we arrive at

$$
\begin{aligned}
(T(u) & -T(v),(u-v)) \\
\quad \leq & \left(\theta_{3} \theta_{4} \theta_{5} \theta_{6} \theta_{7} \theta_{8}+\theta_{1} \theta_{2}+\omega_{1} \omega_{2}+1\right)\|u-v\|^{2} .
\end{aligned}
$$

Since it is assumed that $u, v$ are bounded in $\mathscr{H}$, we can obviously obtain the following positive constant $M$ satisfying

$$
\|u-v\| \leq 2 M^{2}
$$

Therefore, we can conclude that

$$
\begin{aligned}
(T(u) & -T(v),(u-v)) \\
& \leq 2 M^{2}\left(\theta_{3} \theta_{4} \theta_{5} \theta_{6} \theta_{7} \theta_{8}+\theta_{1} \theta_{2}+\omega_{1} \omega_{2}+1\right)\|u-v\|
\end{aligned}
$$

taking here

$$
F=2 M^{2}\left(\theta_{3} \theta_{4} \theta_{5} \theta_{6} \theta_{7} \theta_{8}+\theta_{1} \theta_{2}+\omega_{1} \omega_{2}+1\right)
$$

and then Hypothesis 1 is verified. We will now verify Hypothesis 2; to do this we quickly compute the relation as follows.

Proof. Consider

$$
\begin{aligned}
(T(u)-T(v), z)= & \left(\partial_{x t}^{2}(u-v), z\right)+\left(u^{2}-v^{2}, z\right) \\
& +((u-v), u-v)+\left(\partial_{x^{2} t^{2}}^{4}\left(u^{2}-v^{2}\right), z\right) .
\end{aligned}
$$

Now, following the discussion presented earlier we obtain

$$
(T(u)-T(v), z) \leq \Phi(C)\|u-v\|\|z\|,
$$

with

$$
\Phi(D)=\left(2 D^{2} f_{3} f_{4} f_{5} f_{6} f_{7} f_{8}+2 D^{2} f_{1} f_{2}+2 D^{2} v_{1} v_{2}+1\right) .
$$

With the above hypothesis proved, we will go ahead with stating the following theorem. 
Theorem 2. Taking into account the initial conditions for (5), then the special solution of (5) $u_{\text {esp }}$ to which $u$ converge is unique.

Proof. Assuming that we can find another special solution, say $v_{\text {esp }}$, then by making use of the inner product together with Hypothesis 1, we have the following:

$$
\left(T\left(u_{\text {esp }}\right)-T\left(v_{\text {esp }}\right),\left(u_{\text {esp }}-v_{\text {esp }}\right)\right) \leq F\left\|u_{\text {esp }}-v_{\text {esp }}\right\|
$$

using the fact that we can find a small natural number $m_{1}$ for which we can find a very small number $\varepsilon$ such respecting the following inequality:

$$
\left\|u_{\text {esp }}-u\right\| \leq \frac{\varepsilon}{2 F}
$$

Also, we can find another natural number $m_{2}$ for which we can find a very small positive number $\varepsilon$ that can respect the fact that

$$
\left\|v_{\mathrm{esp}}-u\right\| \leq \frac{\varepsilon}{F 2}
$$

taking therefore $m=\max \left(m_{1}, m_{2}\right)$; we have without fear that

$$
\begin{aligned}
& \left(T\left(u_{\text {esp }}\right)-T\left(v_{\text {esp }}\right),\left(u_{\text {esp }}-v_{\text {esp }}\right)\right) \\
& \quad \leq F\left\|u_{\text {esp }}-v_{\text {esp }}\right\|=F\left\|u_{\text {esp }}-u+u-v_{\text {esp }}\right\| .
\end{aligned}
$$

Making use of the triangular inequality, we obtain the following:

$$
\begin{aligned}
& \left(T\left(u_{\text {esp }}\right)-T\left(v_{\text {esp }}\right),\left(u_{\text {esp }}-v_{\text {esp }}\right)\right) \\
& \quad \leq F\left(\left\|u_{\text {esp }}-u\right\|+\left\|v_{\text {esp }}-u\right\|\right) \leq \varepsilon
\end{aligned}
$$

It therefore turns out that

$$
\left(T\left(u_{\text {esp }}\right)-T\left(v_{\text {esp }}\right),\left(u_{\text {esp }}-v_{\text {esp }}\right)\right)=0 .
$$

But according to the law of the inner product, the above equation implies that

$$
T\left(u_{\text {esp }}\right)-T\left(v_{\text {esp }}\right)=0 \text { or } \quad\left(u_{\text {esp }}-v_{\text {esp }}\right)=0 .
$$

This concludes the uniqueness of our special solution.

\section{Application of the Proposed Method}

We will present in this section the application of this method for (5) since the local convergence and uniqueness have been presented.

Consider

$$
\partial_{x t}^{2} u+u^{2}+u+\partial_{x^{2} t^{2}}^{4} u^{2}=0 .
$$

According to the proposed method, we have that

$$
\begin{aligned}
& u(x, t) \\
& \quad=G(x, t) \\
& \quad+\int_{0}^{x} \int_{0}^{t} \lambda(\rho, \tau)\left[\partial_{\rho \tau}^{2} u+u^{2}+u+\partial_{\rho^{2} \tau^{2}}^{4} u^{2}\right] d \rho d \tau .
\end{aligned}
$$

The method has been modified into an iteration method [48 ] in the following approach; its correction functional can be written down as follows:

$$
\begin{aligned}
u_{n+1}(x, t) & \\
= & G(x, t) \\
& +\int_{0}^{x} \int_{0}^{t} \lambda(\rho, \tau)\left[\partial_{\rho \tau}^{2} u+\overline{u^{2}}+u+\partial_{\rho^{2} \tau^{2}}^{4} \overline{u^{2}}\right] d \rho d \tau .
\end{aligned}
$$

$\tilde{u}(x, t)$ is pondered as a circumscribed adaptation meaning $\delta \tilde{u}(x, t)=0$; therefore we can by applying integration by part in both directions $x$ - $t$ obtain

$$
\partial_{x t}^{2} \lambda+\lambda=0
$$

for which the solution

$$
\lambda(x, t)=\operatorname{Cosh}(-x+t)
$$

with the above Lagrange multiplier; we can set the iteration formula as

$$
\begin{aligned}
u_{n+1}(x, t)= & u_{n} \\
+\int_{0}^{x} \int_{0}^{t} & \operatorname{Cosh}(-\rho+\tau) \\
& \quad \times\left[\partial_{\rho \tau}^{2} u_{n}+u_{n}^{2}+u_{n}+\partial_{\rho^{2} \tau^{2}}^{4} u_{n}^{2}\right] d \rho d \tau
\end{aligned}
$$

with initial guess $u_{0}=G(x, t)$ where

$$
u(x, t)=\lim _{n \rightarrow \infty} u_{n+1}(x, t) .
$$

We can resume the above process in the following algorithm.

Algorithm 3. Consider the following:

(i) Input: $G(x, t)$ as initial guest.

(ii) $j$-number terms in the rough calculation.

(iii) Output: $u_{\text {approx }}(x, t)$, the approximate solution.

Step 1. Put $u_{0}(x, t)=G(x, t)$ and $u_{\text {approx }}(x, t)=u_{0}(x, t)$,

Step 2. For $j=0$ to $n-1$, do Step 3, Step 4, and Step 5 .

Step 3. Compute

$$
v_{n}=\int_{0}^{x} \int_{0}^{t} \operatorname{Cosh}(-\rho+\tau)\left[\partial_{\rho \tau}^{2} u_{n}+u_{n}^{2}+u_{n}+\partial_{\rho^{2} \tau^{2}}^{4} u_{n}^{2}\right] d \rho d \tau .
$$

Step 4. Compute

$$
u_{n+1}(x, t)=v_{n}+u_{n} \text {. }
$$

Step 5. Compute $u_{\text {approx }}(x, t)=u_{\text {approx }}(x, t)+u_{n+1}(x, t)$. Stop. 
4.1. Special Solution. We will in this subsection make use of the proposed algorithm to present the special solution.

We assume that the initial guest is given by

$$
G(x, t)=1 ;
$$

then using the iteration formula, we obtain the following:

$$
\begin{aligned}
& u_{1}(x, t)=\operatorname{Cosh}[x]-\operatorname{Cosh}[t-x]+\operatorname{Cosh}[x] \\
& u_{2}[x, t]=\operatorname{Cosh}[t]-\operatorname{Cosh}[t-x]+\operatorname{Cosh}[x] \\
& +\frac{1}{72}(-355+108 t x+366 \operatorname{Cosh}[t] \\
& -21 \operatorname{Cosh}[2 t]+10 \operatorname{Cosh}[3 t] \\
& -6 \operatorname{Cosh}[t-3 x]+30 \operatorname{Cosh}[2 t-3 x] \\
& +18 \operatorname{Cosh}[t-2 x]+30 \operatorname{Cosh}[3 t-2 x] \\
& -414 \operatorname{Cosh}[t-x]-27 \operatorname{Cosh}[2(t-x)] \\
& -34 \operatorname{Cosh}[3(t-x)]+18 \operatorname{Cosh}[2 t-x] \\
& -6 \operatorname{Cosh}[3 t-x]+366 \operatorname{Cosh}[x] \\
& -21 \operatorname{Cosh}[2 x]+10 \operatorname{Cosh}[3 x] \\
& +36 \operatorname{Cosh}[t+x]-216 x \operatorname{Sinh}[t] \\
& +36 x \operatorname{Sinh}[2 t]-216 t \operatorname{Sinh}[x] \\
& +36 t \operatorname{Sinh}[2 x]) \\
& u_{3}(x, t)=\operatorname{Cosh}[t]-\operatorname{Cosh}[t-x]+\operatorname{Cosh}[x] \\
& +\frac{1}{72}(-355+108 t x+366 \operatorname{Cosh}[t] \\
& -21 \operatorname{Cosh}[2 t]+10 \operatorname{Cosh}[3 t] \\
& -6 \operatorname{Cosh}[t-3 x]+30 \operatorname{Cosh}[2 t-3 x] \\
& +18 \operatorname{Cosh}[t-2 x]+30 \operatorname{Cosh}[3 t-2 x] \\
& -414 \operatorname{Cosh}[t-x]-27 \operatorname{Cosh}[2(t-x)] \\
& -34 \operatorname{Cosh}[3(t-x)]+18 \operatorname{Cosh}[2 t-x] \\
& -6 \operatorname{Cosh}[3 t-x]+366 \operatorname{Cosh}[x] \\
& -21 \operatorname{Cosh}[2 x]+10 \operatorname{Cosh}[3 x] \\
& +36 \operatorname{Cosh}[t+x]-216 x \operatorname{Sinh}[t] \\
& +36 x \operatorname{Sinh}[2 t]-216 t \operatorname{Sinh}[x] \\
& +36 t \operatorname{Sinh}[2 x]+F(x, t)) \\
& u_{4}(x, t)=\operatorname{Cosh}[t]-\operatorname{Cosh}[t-x]+\operatorname{Cosh}[x] \\
& +\frac{1}{72}(-355+108 t x+366 \operatorname{Cosh}[t] \\
& -21 \operatorname{Cosh}[2 t]+10 \operatorname{Cosh}[3 t] \\
& -6 \operatorname{Cosh}[t-3 x]+30 \operatorname{Cosh}[2 t-3 x]
\end{aligned}
$$

$$
\begin{aligned}
& +18 \operatorname{Cosh}[t-2 x]+30 \operatorname{Cosh}[3 t-2 x] \\
& -414 \operatorname{Cosh}[t-x]-27 \operatorname{Cosh}[2(t-x)] \\
& -34 \operatorname{Cosh}[3(t-x)]+18 \operatorname{Cosh}[2 t-x] \\
& -6 \operatorname{Cosh}[3 t-x]+366 \operatorname{Cosh}[x] \\
& -21 \operatorname{Cosh}[2 x]+10 \operatorname{Cosh}[3 x] \\
& +36 \operatorname{Cosh}[t+x]-216 x \operatorname{Sinh}[t] \\
& +36 x \operatorname{Sinh}[2 t]-216 t \operatorname{Sinh}[x] \\
& +36 t \operatorname{Sinh}[2 x]+F(x, t)+H(x, t)) .
\end{aligned}
$$

In this case, we consider the small natural number $m$ to be 4 such that the special solution gives

$$
\begin{aligned}
u_{\text {esp }}(x, t)=\operatorname{Cosh}[t] & -\operatorname{Cosh}[t-x]+\operatorname{Cosh}[x] \\
+\frac{1}{72} & (-355+108 t x+366 \operatorname{Cosh}[t] \\
& -21 \operatorname{Cosh}[2 t]+10 \operatorname{Cosh}[3 t] \\
& -6 \operatorname{Cosh}[t-3 x]+30 \operatorname{Cosh}[2 t-3 x] \\
& +18 \operatorname{Cosh}[t-2 x]+30 \operatorname{Cosh}[3 t-2 x] \\
& -414 \operatorname{Cosh}[t-x]-27 \operatorname{Cosh}[2(t-x)] \\
& -34 \operatorname{Cosh}[3(t-x)]+18 \operatorname{Cosh}[2 t-x] \\
& -6 \operatorname{Cosh}[3 t-x]+366 \operatorname{Cosh}[x] \\
& -21 \operatorname{Cosh}[2 x]+10 \operatorname{Cosh}[3 x] \\
& +36 \operatorname{Cosh}[t+x]-216 x \operatorname{Sinh}[t] \\
& +36 x \operatorname{Sinh}[2 t]-216 t \operatorname{Sinh}[x] \\
& +36 t \operatorname{Sinh}[2 x]+F(x, t)+H(x, t)) .
\end{aligned}
$$

We present the graphical representation of the special solution of (5) in Figure 1.

Example 4. Let us consider the following partial differential equation:

$$
\begin{gathered}
\partial_{x t}^{2} u(x, t)+u(x, t)=0, \\
u(x, 0)=g(x), \quad u(0, t)=h(t) .
\end{gathered}
$$

Employing the methodology of the proposed method, we obtain the following Lagrange multiplier:

$$
\lambda(x, t)=-1 \text {. }
$$

Then, the iteration method is given by

$$
u_{n+1}(x, t)=u_{n}+\int_{0}^{x} \int_{0}^{t}\left[\partial_{\rho \tau}^{2} u_{n}+u_{n}\right] d \rho d \tau
$$

choosing the initial guest to be

$$
u_{0}(x, t)=1-x y .
$$




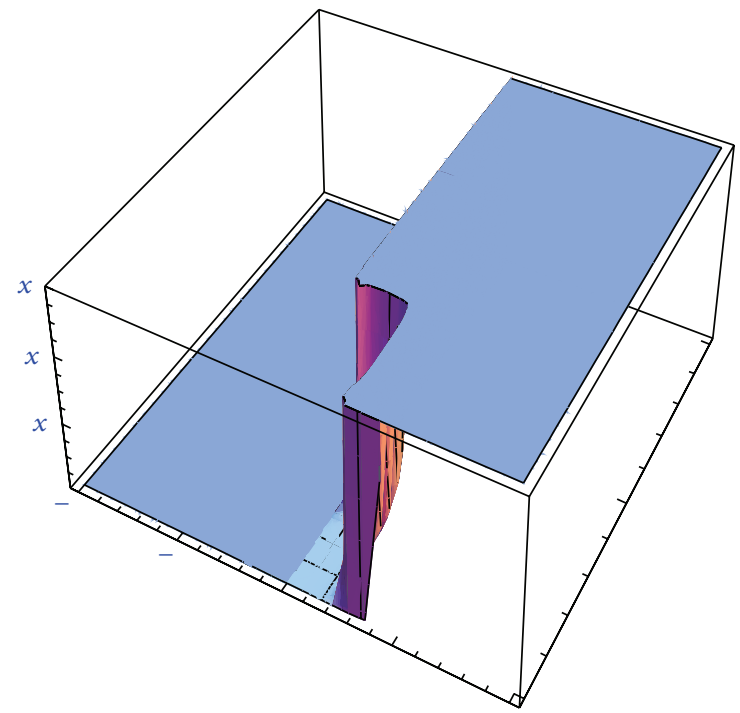

FIGURE 1: Special solution for $m=4$.

Using the algorithm associate to the iteration formula (49), we obtain

$$
\begin{aligned}
& u_{7}=1+\frac{x^{2}}{2}+\frac{x^{4}}{24}+\frac{x^{6}}{720}+\frac{x^{8}}{40320} \\
& +t\left(-x-\frac{x^{3}}{6}-\frac{x^{5}}{120}-\frac{x^{7}}{5040}\right) \\
& +t^{3}\left(-\frac{x}{6}-\frac{x^{3}}{36}-\frac{x^{5}}{720}-\frac{x^{7}}{30240}\right) \\
& +t^{5}\left(-\frac{x}{120}-\frac{x^{3}}{720}-\frac{x^{5}}{14400}-\frac{x^{7}}{604800}\right) \\
& +t^{7}\left(-\frac{x}{5040}-\frac{x^{3}}{30240}\right. \\
& \left.-\frac{x^{5}}{604800}-\frac{x^{7}}{25401600}\right) \\
& +t^{8}\left(\frac{1}{40320}+\frac{x^{2}}{80640}+\frac{x^{4}}{967680}\right. \\
& \left.+\frac{x^{6}}{29030400}+\frac{x^{8}}{1625702400}\right) \\
& +t^{6}\left(\frac{1}{720}+\frac{x^{2}}{1440}+\frac{x^{4}}{17280}\right. \\
& \left.+\frac{x^{6}}{518400}+\frac{x^{8}}{29030400}\right)
\end{aligned}
$$

$$
\begin{aligned}
& +t^{4}\left(\frac{1}{24}+\frac{x^{2}}{48}+\frac{x^{4}}{576}+\frac{x^{6}}{17280}+\frac{x^{8}}{967680}\right) \\
& +t^{2}\left(\frac{1}{2}+\frac{x^{2}}{4}+\frac{x^{4}}{48}+\frac{x^{6}}{1440}+\frac{x^{8}}{80640}\right) \\
& u_{9}(x, t)=1+\frac{x^{2}}{2}+\frac{x^{4}}{24} \\
& +\frac{x^{6}}{720}+\frac{x^{8}}{40320}+\frac{x^{10}}{3628800} \\
& +t\left(-x-\frac{x^{3}}{6}-\frac{x^{5}}{120}-\frac{x^{7}}{5040}-\frac{x^{9}}{362880}\right) \\
& +t^{3}\left(-\frac{x}{6}-\frac{x^{3}}{36}-\frac{x^{5}}{720}\right. \\
& \left.-\frac{x^{7}}{30240}-\frac{x^{9}}{2177280}\right) \\
& +t^{5}\left(-\frac{x}{120}-\frac{x^{3}}{720}-\frac{x^{5}}{14400}\right. \\
& \left.-\frac{x^{7}}{604800}-\frac{x^{9}}{43545600}\right) \\
& +t^{7}\left(-\frac{x}{5040}-\frac{x^{3}}{30240}-\frac{x^{5}}{604800}\right. \\
& \left.-\frac{x^{7}}{25401600}-\frac{x^{9}}{1828915200}\right) \\
& +t^{9}\left(-\frac{x}{362880}-\frac{x^{3}}{2177280}-\frac{x^{5}}{43545600}\right. \\
& \left.-\frac{x^{7}}{1828915200}-\frac{x^{9}}{131681894400}\right) \\
& +t^{10}\left(\frac{1}{3628800}+\frac{x^{2}}{7257600}+\frac{x^{4}}{87091200}\right. \\
& +\frac{x^{6}}{2612736000}+\frac{x^{8}}{146313216000} \\
& \left.+\frac{x^{10}}{13168189440000}\right) \\
& +t^{8}\left(\frac{1}{40320}+\frac{x^{2}}{80640}\right. \\
& +\frac{x^{4}}{967680}+\frac{x^{6}}{29030400} \\
& \left.+\frac{x^{8}}{1625702400}+\frac{x^{10}}{146313216000}\right) \\
& +t^{6}\left(\frac{1}{720}+\frac{x^{2}}{1440}+\frac{x^{4}}{17280}+\frac{x^{6}}{518400}\right. \\
& \left.+\frac{x^{8}}{29030400}+\frac{x^{10}}{2612736000}\right)
\end{aligned}
$$




$$
\begin{array}{r}
+t^{4}\left(\frac{1}{24}+\frac{x^{2}}{48}+\frac{x^{4}}{576}+\frac{x^{6}}{17280}\right. \\
\left.+\frac{x^{8}}{967680}+\frac{x^{10}}{87091200}\right) \\
+t^{2}\left(\frac{1}{2}+\frac{x^{2}}{4}+\frac{x^{4}}{48}+\frac{x^{6}}{1440}\right. \\
\left.+\frac{x^{8}}{80640}+\frac{x^{10}}{7257600}\right) .
\end{array}
$$

Indeed $u_{9}(x, t)$ is Maclaurin series of $\operatorname{Cosh}(x-t)$ of order 10 . Therefore, the exact solution of (47) is

$$
u(x, t)=\lim _{n \rightarrow \infty} u_{n}(x, t)=\operatorname{Cosh}(x-t) .
$$

\section{Conclusion}

Attention has not been paid to the class of partial differential equations with mixed derivatives only. But this class of partial differential equations is used to describe several physical occurrences or real world problems. More importantly, the nonlinear partial differential equations with mixed derivatives only cannot be handled with the commonly used analytical methods. Even some numerical methods [15] that have been recognized as efficient methods cannot handle these nonlinear partial differential equations. Based upon the Lagrange multiplier in two-dimensional space, we proposed an iteration analytical method to solve a class of partial differential equations that could be handled via usual methods including the Laplace transform, Fourier transform, Mellin transform, the Green function, and the Sumudu transform on one hand and on the other hand iteration methods like normal variational iteration method, the normal homotopy perturbation method, the normal homotopy decomposition method, and other methods like perturbation methods. A detailed analysis of convergence and uniqueness was presented. An algorithm showing the resume of the method for solving this example was proposed. The method is highly efficient, easier to use, and also very accurate.

\section{Conflict of Interests}

The authors declare that there is no conflict of interests for this paper.

\section{Authors' Contribution}

Abdon Atangana wrote the first draft. Both authors revised and corrected the final version.

\section{Acknowledgments}

The authors would like to thank the editor and anonymous reviewers for their valuable suggestions toward the enhancement of this paper. Abdon Atangana would like to thank the Claude Leon Foundation for their scholarship.

\section{References}

[1] A. Babakhani and R. S. Dahiya, "Systems of multi-dimensional Laplace transforms and a heat equation," in Proceedings of the 16th Conference on Applied Mathematics, vol. 7 of Electronic Journal of Differential Equations, pp. 25-36, University of Central Oklahoma, Edmond, Okla, USA, 2001.

[2] G. L. Lamb Jr., Introductory Applications of Partial Differential Equations with Emphasis on Wave Propagation and Diffusion, John Wiley \& Sons, New York, NY, USA, 1995.

[3] A. Atangana, "A note on the triple Laplace transform and its applications to some kind of third-order differential equation," Abstract and Applied Analysis, vol. 2013, Article ID 769102, 10 pages, 2013.

[4] A. D. Polyanin and A. V. Manzhirov, Handbook of Integral Equations, CRC Press, Boca Raton, Fla, USA, 1998.

[5] R. N. Bracewell, The Fourier Transform and Its Applications, McGraw-Hill, Boston, Mass, USA, 3rd edition, 2000.

[6] E. U. Condon, "Immersion of the Fourier transform in a continuous group of functional transformations," Proceedings of the National Academy of Sciences of the USA, vol. 23, pp. 158-164, 1937.

[7] G. K. Watugala, "Sumudu transform: a new integral transform to solve differential equations and control engineering problems," International Journal of Mathematical Education in Science and Technology, vol. 24, no. 1, pp. 35-43, 1993.

[8] S. Weerakoon, "Application of Sumudu transform to partial differential equations," International Journal of Mathematical Education in Science and Technology, vol. 25, no. 2, pp. 277-283, 1994.

[9] A. Atangana and A. Kılıçman, "The use of Sumudu transform for solving certain nonlinear fractional heat-like equations," Abstract and Applied Analysis, vol. 2013, Article ID 737481, 12 pages, 2013.

[10] A. Atangana, "Drawdown in prolate spheroidal-spherical coordinates obtained via Green's function and perturbation methods," Communications in Nonlinear Science and Numerical Simulation, vol. 19, no. 5, pp. 1259-1269, 2014.

[11] A. Atangana, "On the singular perturbations for fractional differential equation," The Scientific World Journal, vol. 2014, Article ID 752371, 9 pages, 2014.

[12] E. Yusufoglu, "Variational iteration method for construction of some compact and noncompact structures of Klein-Gordon equations," International Journal of Nonlinear Sciences and Numerical Simulation, vol. 8, no. 2, pp. 153-158, 2007.

[13] G.-C. Wu and D. Baleanu, "Variational iteration method for the Burgers' flow with fractional derivatives: new Lagrange multipliers," Applied Mathematical Modelling: Simulation and Computation for Engineering and Environmental Systems, vol. 37, no. 9, pp. 6183-6190, 2013.

[14] H. Jafari and V. Daftardar-Gejji, "Solving a system of nonlinear fractional differential equations using Adomian decomposition," Journal of Computational and Applied Mathematics, vol. 196, no. 2, pp. 644-651, 2006. 
[15] H. Vazquez-Leal, A. Sarmiento-Reyes, Y. Khan, U. FilobelloNino, and A. Diaz-Sanchez, "Rational biparameter homotopy perturbation method and Laplace-Padé coupled version," Journal of Applied Mathematics, vol. 2012, Article ID 923975, 21 pages, 2012.

[16] Y.-G. Wang, W.-H. Lin, and N. Liu, "A homotopy perturbationbased method for large deflection of a cantilever beam under a terminal follower force," International Journal for Computational Methods in Engineering Science and Mechanics, vol. 13, no. 3, pp. 197-201, 2012.

[17] Z. Šmarda and Y. Khan, "Singular initial value problem for a system of integro-differential equations," Abstract and Applied Analysis, vol. 2012, Article ID 918281, 18 pages, 2012.

[18] A. Atangana and A. Secer, "The time-fractional coupledKorteweg-de-Vries equations," Abstract and Applied Analysis, vol. 2013, Article ID 947986, 8 pages, 2013. 


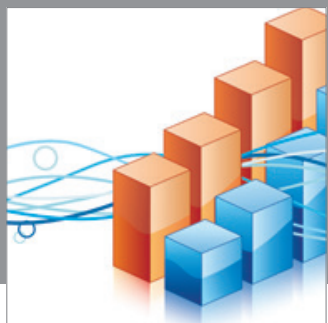

Advances in

Operations Research

mansans

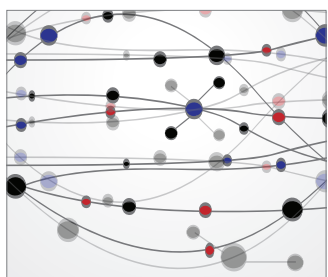

The Scientific World Journal
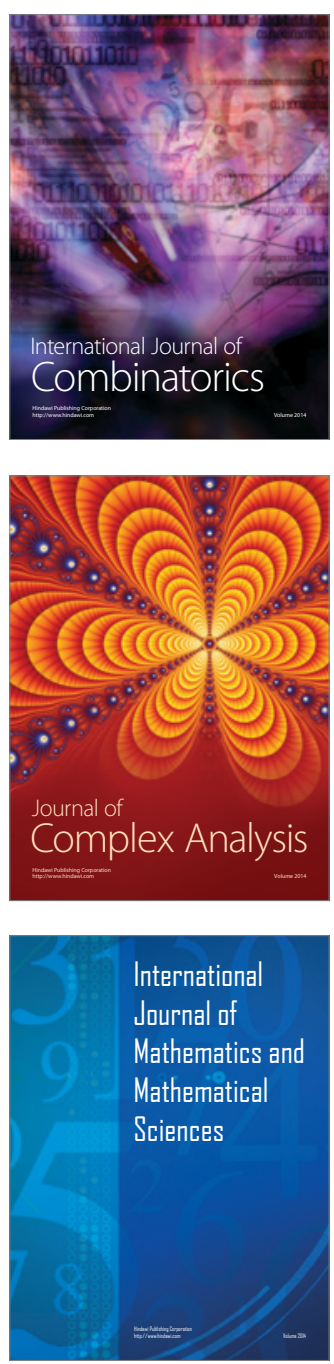
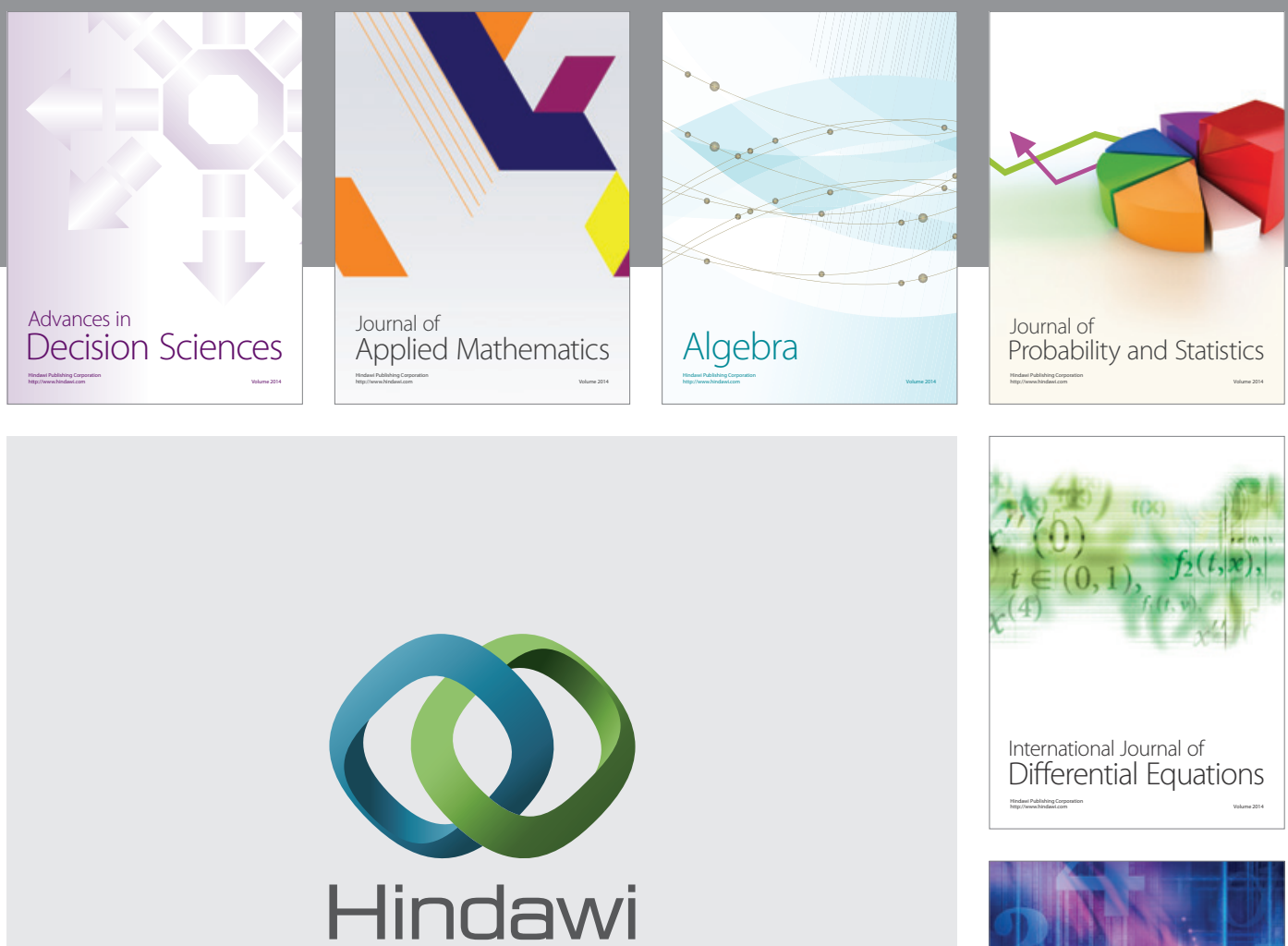

Submit your manuscripts at http://www.hindawi.com
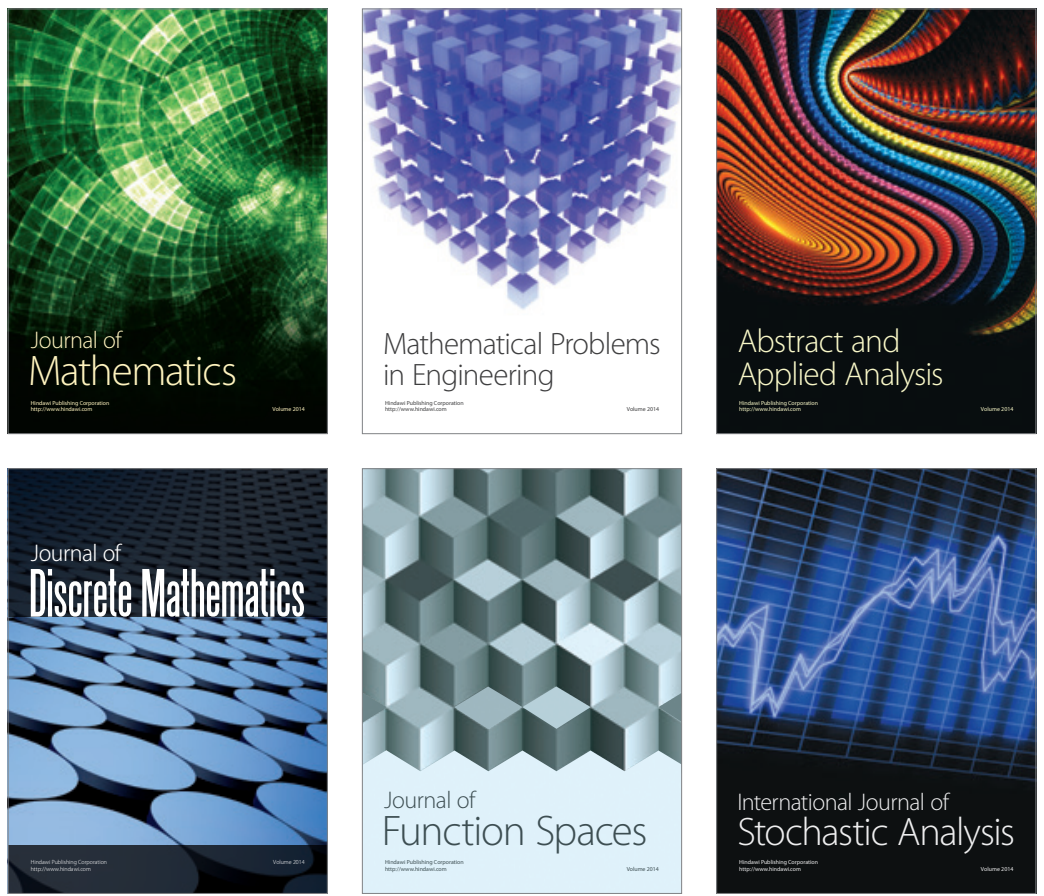

Journal of

Function Spaces

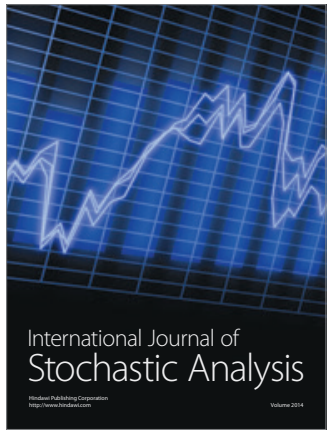

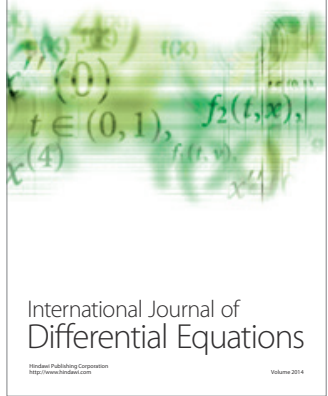
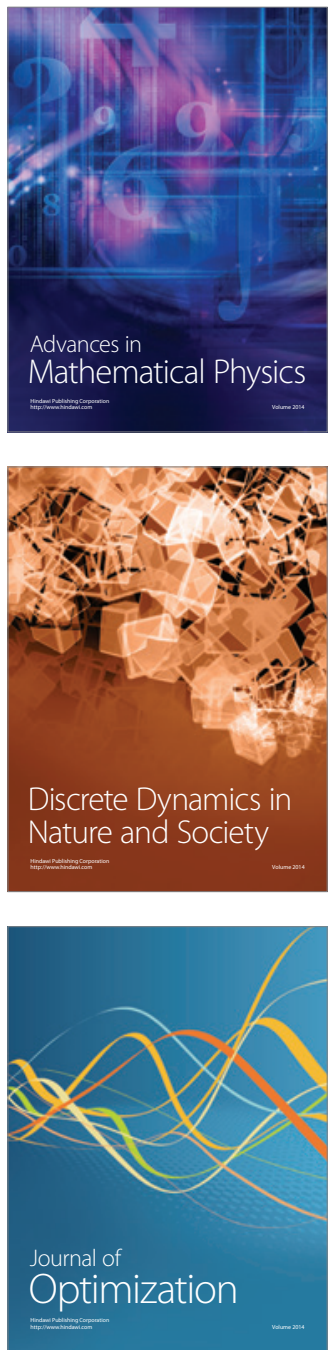